\title{
The efficiency of FLAIR images for hemodynamic change after STA-MCA bypass with Moyamoya disease and symptomatic steno-occlusive disorder
}

Moon Sun Park

Seung Young Jung, Se Hun Chang, Jae Gook Kim*

Department of Neurosurgery and Neurology*, Eulji University Hospital, College of Medicine, Eulji University, Daejeon 302-799, Korea.

\section{INTRODUCTION}

MR perfusion and SPECT are well known imaging studies to evaluate hemodynamic change between prior to and following STA-MCA anastomosis. But their side effects and invasiveness make discomfort to patients. We evaluated the "Ivy sign" and "spaghetti sign" on MR FLAIR images in adult patients with Moyamoya disease and steno-occlusive disorder performed STA-MCA bypass anastomosis and compared it with result of SPECT and MR perfusion

inthyountense vessels

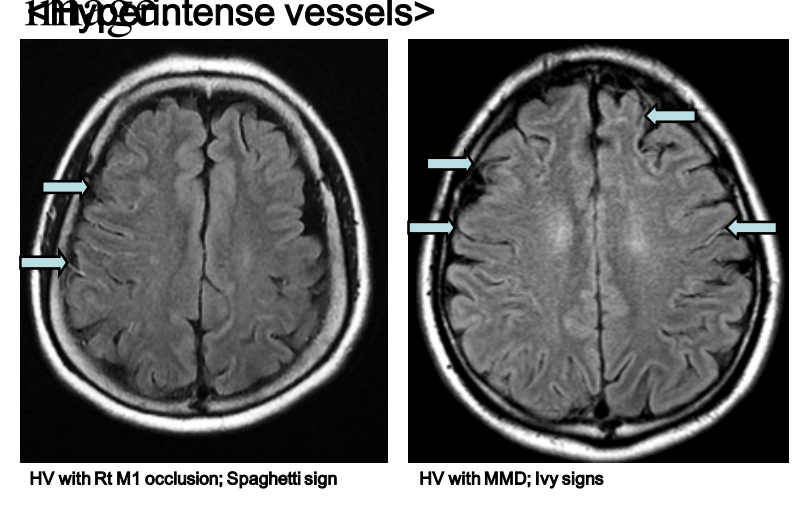

Hyperintense v
sign ) on post-cor
highly with exi
Assumed to be
sign. However,
correlation bet
in patients with

We enrolled forty-nine patients who were diagnosed ICA steno-occlusive disorder and Moyamoya disease and underwent STA-MCA anastomosis at our center during 4 years. The presence of the Ivy sign and spaghetti sign on MR FLAIR images was classified as Negative (0), Minimal (1), and Positive (2). Regions were classified into four territories: ACA, anterior MCA, posterior MCA and PCA. Correlations between FLAIR images and hemodynamic status measured by

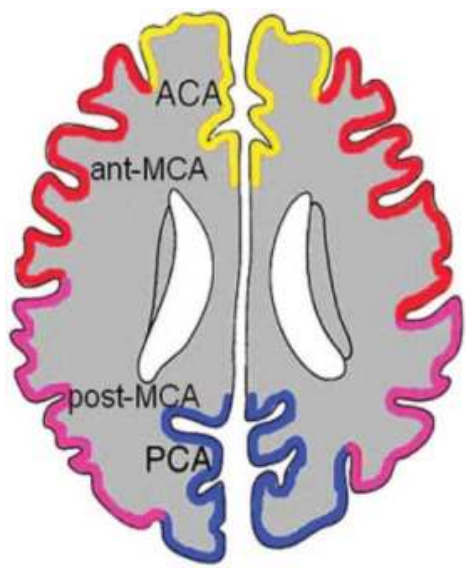
SPECT were evaluated.

\section{RESULTS Before SURGERY}

Among 49 patients, 17 patients were treated with moyamoya disease, and 32 patients were symptomatic steno-occlusive disorder. Minimal and Positive Ivy and Spaghetti sign was seen in $36(74 \%)$ of 49 patients.

Focal or global decrease of resting CBF on perfusion MRI in $92 \%$ of lesions and $83 \%$ of area of positive or minimal HV exhibited lower CVR on SPECT and Frequency of positive HV and its score (Ivy score) were highest in post MCA region in Moyamoya disease, and Spaghetti score highest in ant MCA region in steno-occlusive disorder.

\section{RESULTS After SURGERY}

In all patients with F/U, FLAIR after 3 to 14 month of surgery. Positive or Minimal HV in 49 patients before surgery disappeared in 21 and decreased in 11 after surgery (65\%). Perfusion MRI and SPECT demonstrated hemodynamic and CVR improvement in area in which HV disappeared or decreased after surgery.

In MMD, Ivy score was improved as below

ACA territory : $10 \rightarrow 5$

Anterior MCA : $17->5$

Posterior MCA : 24 -> 11

PCA : 3->3

In symptomatic steno-occlusive disorder, Spaghetti score also improved ACA territory : $12 \rightarrow 7$

Anterior MCA : $23->10$

Posterior MCA : 7 -> 4

PCA : 3->2

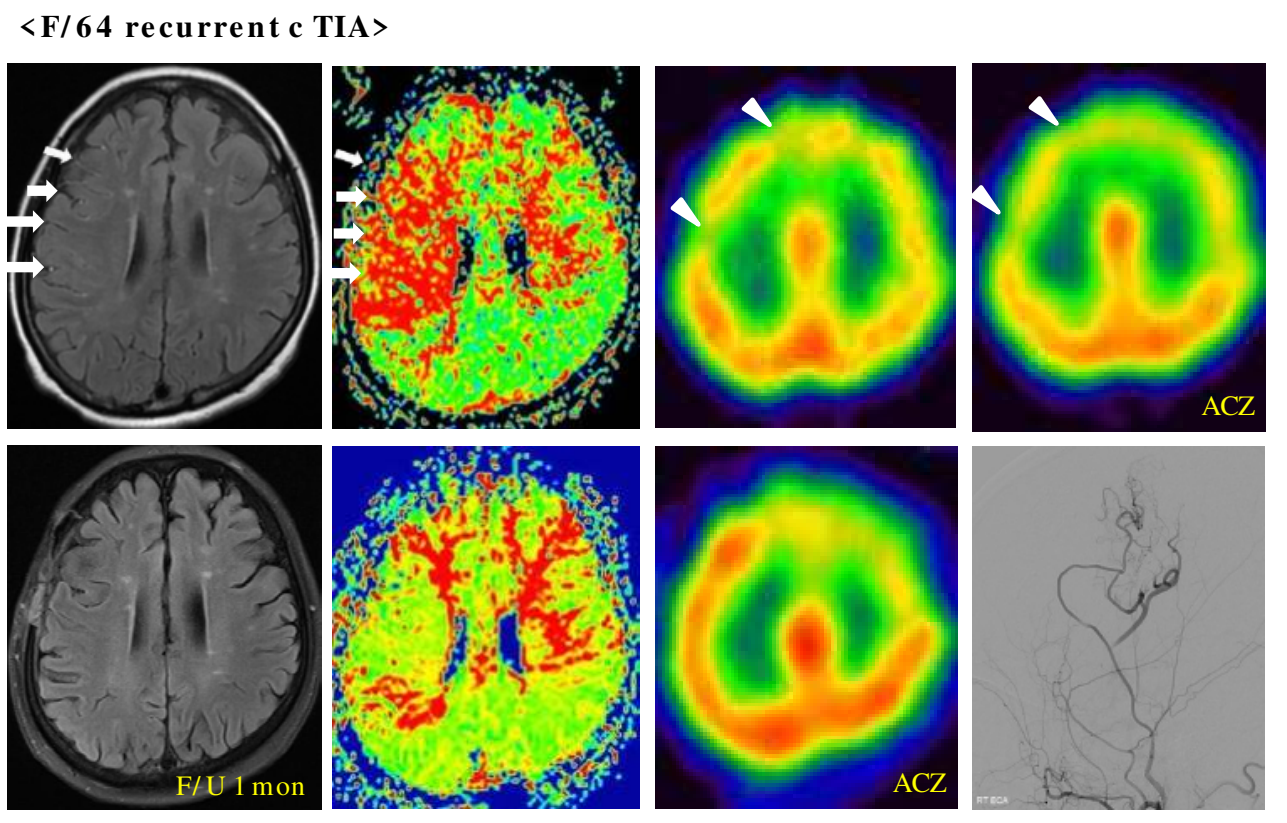

\section{DISCUSSION}

According to recent studies, the ivy sign on MR FLAIR images showed a new diagnostic value in detecting brain hemodynamic changes. MR FLAIR images in evaluating brain hemodynamic changes, further studies have been conducted to examine whether it is also a useful indicator at a follow-up in patients who underwent STA-MCA anastomosis.

Change of Hyperintense vessels mean Slow antegrade and retrograde collateral flows are corresponding to hyperintense vessels. Ivy sign and Spaghetti sign on FLAIR in affected hemisphere. Revealed revascularization effective for decreasing $\mathrm{HV}$ after $\mathrm{F} / \mathrm{U}$ and decrease of $\mathrm{HV}$ on bypass-established hemisphere associated with improved hemodynamic status. Presence of $\mathrm{HV}$ revealed to be marker of hemodynamic status before and after surgery

\section{CONCLUSSION}

After STA-MCA anastomosis, ivy signs and spaghetti signs were decreased in the cerebral hemisphere. As compared with conventional diagnostic modalities such as SPECT and MR perfusion images, the ivy sign and spaghetti sign on MR FLAIR is considered as a useful indicator in detecting brain hemodynamic changes between preoperatively and postoperatively in Moyamoya disease and symptomatic steno-occlusive disorders. 